\title{
Contact Damage Produced in Si and MgO by Nanoindentation
}

\author{
B.J. Hockey \\ Ceramics Divison, National Institute of Standards and Technology \\ Gaithersburg, MD 20899
}

Plane-section TEM has been used to characterize residual defect structures produced in $\{111\} \mathrm{Si}$ and $\{001\} \mathrm{MgO}$ by Berkovich nanoindentation at loads ranging from $5 \mathrm{mN}$ to $0.1 \mathrm{mN}$. The results provide a comparison of two materials known to exhibit different elastic and plastic behavior, but comparable hardness values. For both materials, the results represent the initial phases of an effort to systematically define the characteristic features, including spatial size, of the deformation that occurs in various brittle materials at ulta-low loads or, alternatively, small penetration depths. At such small penetration depths or loads, direct evidence on active deformation processes, stress thresholds for activation of deformation processes (including phase transformations) and related defects, such as cracks, is non-existent for $\mathrm{Si}$ and other brittle materials. Representative near- [001] zone axis views of $0.1 \mathrm{mN}, 1 \mathrm{mN}$ and $5 \mathrm{mN}$ indentations in $\mathrm{MgO}$ are shown in figure $1 \mathrm{a}$ a-c, respectively. Regardless of load, the deformation can be described in terms of slip on the expected $<110>\{110\}$ slip systems for MgO. For loads of $1 \mathrm{mN}$ and less (Fig. 1 a and b), slip is found to occur primarily on the four $\{110\}_{45}$ planes that are inclined to the (001) surface and only limitedly on the pair of $\{110\}_{90}$ planes normal to the surface. Most dislocations on $\{110\}_{45}$ are in the form of half-loops that extend radially from the area of contact. At $0.1 \mathrm{mN}$ (Fig. 1a), the small number of dislocations (again, primarily on the inclined $\{110\}_{45}$ planes) appears to represent the initial stages of plastic deformation during contact. At $5 \mathrm{mN}$, there is a significant increase in dislocation density within the central region of the residual defect structure, providing an outer limit to the area of contact, as well as radially extending arrays of dislocations on $\{110\}_{90}$. Comparative views of $0.25 \mathrm{mN}, 1 \mathrm{mN}$ and $5 \mathrm{mN}$ indentations in $\{111\} \mathrm{Si}$ are shown in Figure $2 \mathrm{a}-$ d. In each case, the indentations are marked by a central, triangular-shaped region, defined either by either by strain or defect contrast, which can be identified as the projected area of contact during indentation with a Berkovich indenter. At $0.25 \mathrm{mN}$ (Fig. 2a), small $(\sim 10-20 \mathrm{~nm})$, dislocation loops are found both within and along the periphery of the area of contact. No evidence for cracks or phase-transformed regions could be found. At $1 \mathrm{mN}$, indentation sites characteristically exhibited a three-fold array of dislocation loops and a three-fold set of cracks, which are totally contained within the area of contact. The location of these cracks suggests that the form along the sharp corners of the pyramidal-shaped indenter. While the three-fold array of dislocations are still under investigation, they appear to correspond to partial dislocation loops on the set of three $\{111\}$ slip planes that are inclined to the (111) surface. Additional HREM observations on one $1 \mathrm{mN}$ indentation provided evidence for the presence of small ( $\sim 50 \mathrm{~nm}$ or less in diameter) phase transformed crystallites contained within the central region of the area of contact. Observations on 5 $\mathrm{mN}$ indentation sites showed sample to sample differences, which related to the presence or absence of an amorphous surface layer within the area of contact. In certain cases, Fig. $1 \mathrm{c}$ and d, the triangular area of contact with the indenter is covered by a relatively thick amorphous phase which, in low magnification views, obscures the presence of the three-fold set of internal cracks similar to those associated with $1 \mathrm{mN}$ indentations (Fig. 2b). In these cases, the indentation sites are also marked by relatively dense arrays of dislocations on the set of three $\{111\}$ slip planes inclined to the (111) surface (Fig. 2d). In other cases, the amorphous surface layer was either absent or markedly reduced in thickness, and the central cracks were clearly defined. In these cases, the 
density of slip dislocations on the inclined $\{111\}$ planes was significantly reduced in comparison to that seen in Fig., $2 \mathrm{c}$ and d. The underlying reasons for these observed differences in the residual structure of $5 \mathrm{mN}$ indentations cannot be explained and are currently under investigation. In discussing the TEM results seen here, particular emphasis will be placed on their value in determining the actual projected area of contact and, consequently, the mean contact stress developed during indentation.
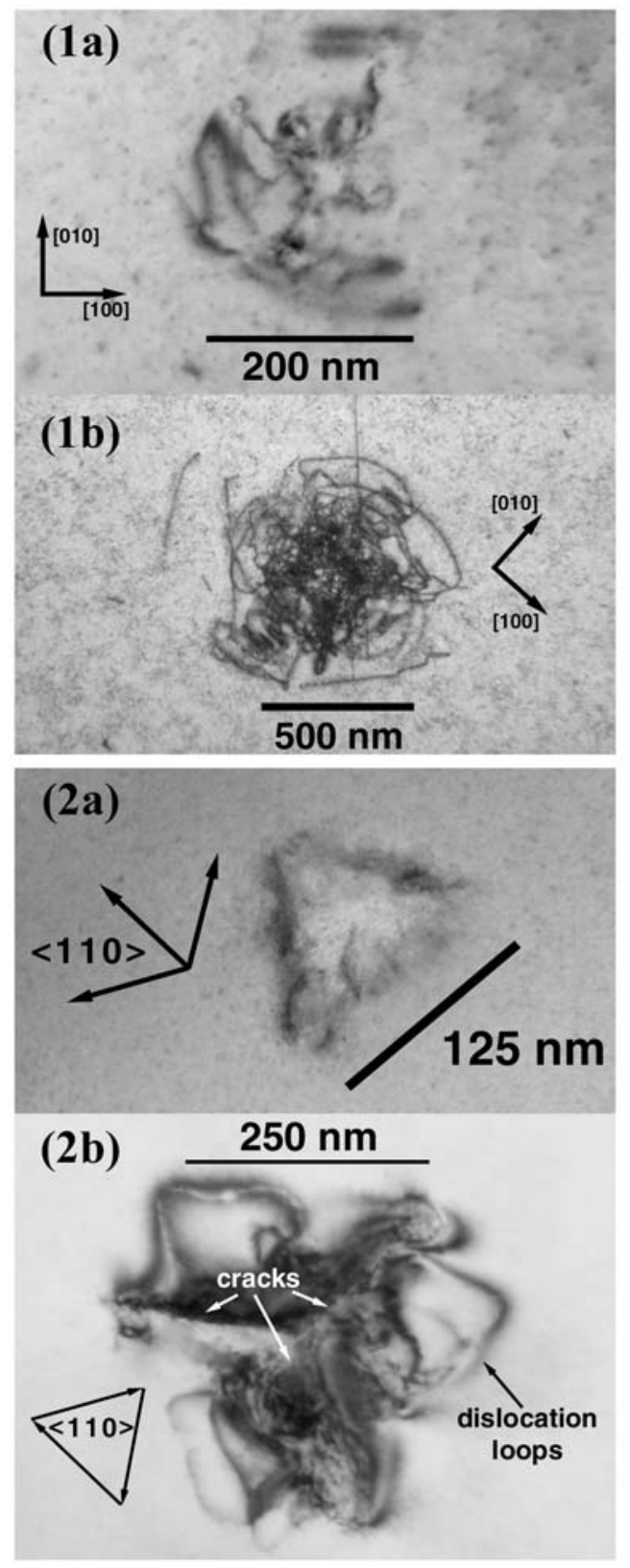

Figure 2. Plane-section TEM views of indentation sites on $\{111) \mathrm{Si}$.

a) $0.25 \mathrm{mN}$ load; b) $1.0 \mathrm{mN}$ load;

c) $5.0 \mathrm{mN}$ load BF, d) same $5 \mathrm{mN}$ in DF

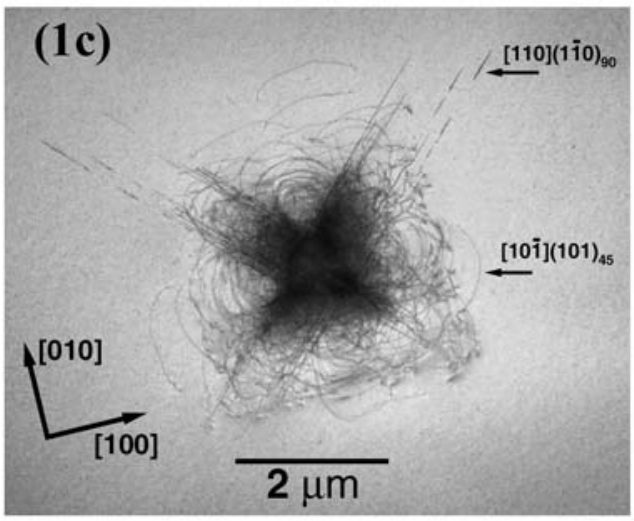

Figure 1. Plane-section TEM views of indentation sites on (001) MgO. a) $0.1 \mathrm{mN}$ load; b) $1.0 \mathrm{mN}$ load; c) $5.0 \mathrm{mN}$ load

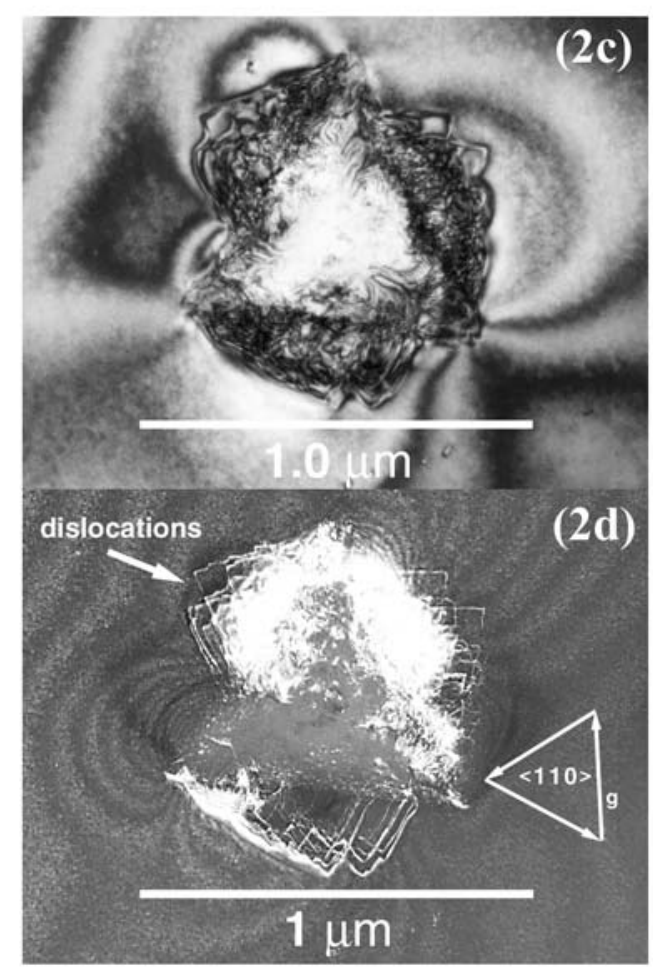

